\title{
Die Stephanusrede Apg. 7: eine Märtyrerapologie.
}

Von Wilhelm Mundle in Marburg.,

Über die Rede, die die Apostelgeschichte dem Protomartyr Stephanus vor seinem Ende in den Mund legt, gibt es eine ausgebreitete Spezialliteratur ${ }^{1}$. Aber wer sich die Mühe macht, sie durchzuarbeiten, wird sie mit einem Gefühl der.Unbefriedigung beiseite legen und finden, dab das hier liegende exegetische Problem noch keineswegs gelöst ist. Denn so wenig Schwierigkeiten im großen und ganzen die Einzelexegese der Rede bereitet, so rätselhaft ist bisher Sinn und Zusammenhang des Ganzen geblieben. So mag es gerechtfertigt erscheinen, wenn hier der Versuch gemacht wird, einen neuen Weg zum Verständnis des vielbehandelten Abschnittes zu suchen. Denn von der richtigen Auffassung der Rede hängt das Urteil über die literarische Komposition der ganzen Stephanusgeschichte sehr wesentlich mit ab. Auf die Auseinandersetzung mit anderen Erklärungsversuchen kann ich im folgenden nur so weit eingehen, als es zur Heraushebung meiner eigenen Auffassung und der für die Untersuchung maßgebenden methodischen Grunđsätze nōtig ist.

Die Exegese hat von der Tatsache auszugehen, daß die Annahme, die Rede sei eine wirklich vom Protomartyr gehaltene Ansprache an den Hohenpriester und das Synedrium, sehr wenig Wahrscheinlichkeit hat. Die allgemeinere Frage, wie es überhaupt mit der Echtheit der Protokolle, die wir in altchristlichen Märtyrerakten finden, bestellt ist, kann hier auf sich beruhen. Sie ist beim Stande der heutigen Forschung noch nicht gelöst und wird sich nur von Fall zu Fall entscheiden lassen. In diesem Falle aber sollte für jeden, der sich nicht auf den Standpunkt der wörtlichen Bibelinspiration zu stellen vermag, die Antwort selbstverständlich sein. $\mathrm{DaB}$ in dem Momente höchster Erregung, in der die Menge den Stephanus, empört über seine Lästerung, vor das Tribunal des Hohenpriesters schleppt - in den Einzelheiten ist die geschilderte Situation nicht völlig deutlich - daß in diesem Augenblick der Hohepriester gewillt gewesen sei, eine Auseinandersetzung des

1 Das Wichtigste davon ist bei Wendt, Die Apostelgeschichte 1913 (Meyers Komm. Bd. III) S. 137 verzeichnet. Hinzuzufügen wäre noch von neueren Untersuchungen die Arbeit von R. Schumacher, Der Diakon Stephanus ( Neutest. Arbeiten, herausg. von Meinertz Wr, 4), die einen guten Einblick in die Geschichte der Exegese gibt. Den Hinweis auf sie verdanke ich Herrn Prof. Schmitz-Münster. 
Stephanus entgegenzunehmen, die auf den eigentlichen Anklagepunkt überhaupt nicht eingeht ${ }^{1}$, wird man als ziemlich un wahrscheinlich bezeichnen können. Und ebensowenig läßt es sich denken, daß Stephanus in einem solchen Augenblick zu derartigen Auseinandersetzungen aufgelegt gewesen wäre. Die Annahme aber, daß irgend einer von den Christen - wenn er etwa zugegen gewesen wäre in dieser Situation protokollarische Aufzeichnungen hätte machen können oder daß den Christen Einsicht in ein amtliches Protokoll möglich gewesen wäre, wird man schon deshalb für sehr fraglich halten müssen, weil ja die ganze Szene überhaupt nicht den Eindruck einer regelrechten Gerichtsverhandlung erweckt. Von diesen Erwägungen aus wird es gerechtfertigt erscheinen, wenn man in der Stephanusrede nicht die Gedanken des ersten Märtyrers, sondern des Verfassers der Apostelgeschichte wiederfinden will.

Man könnte hiergegen einwenden, daß die Möglichkeit bestände, zwar nicht in der Rede eine Wiedergabe der in der Todesstunde gehaltenen Apologie des Stephanus, aber doch wirkliches Gedankengut desselben zu finden, das dem Verfasser der Acta durch irgendwelche Überlieferung übermittelt und von $\mathrm{ihm}$ verwertet worden sei. Diese Annahme kann allerdings nicht von vornherein als undenkbar bezeichnet werden, aber es bleibt doch zum mindesten fraglich, ob wir uns den Lukas ${ }^{2}$ nach Analogie eines modernen, verschiedene Quellen kombinierenden Historikers (trotz Lc I $_{1}$ ff.) vorzustellen haben. Im Texte selbst hat diese Vermutung keinen Anhalt und die Beweislast für sie darf deshalb demjenigen zugeschoben werden, der für sie eintritt. $\mathrm{Er}$ hätte nachzuweisen, daß in der Rede Anschauungen $\mathrm{zu}$ Worte kommen und Material verwertet ist, das sich von dem, was wir aus der Apostelgeschichte als spézifisch lukanisch erschließen können, mit Deutlichkeit abhebt. Derselbe Beweis würde auch für Stil und Ausdrucksweise zu führen sein. Ehe er erbracht worden ist, und es ist bei der Natur der Dinge kaum $z u$ erwarten, daß er je erbracht werden wird, werden wir zu der Annahme berechtigt sein, daß der Verfasser der Acta selbst aus dieser Darstellung zu uns redet und hier wie in anderen Fällen von dem Stilgesetz der Antike Gebrauch gemacht hat, das dem Schriftsteller erlaubt, durch den Mund seines Helden zu seinen Lesern

1 Wir werden noch sehen, daß das aucl Act $7_{4}$ s nicht der Fall ist.

2 Wenn der Verfasser der Apostelgeschichte im folgenden der Kürze halber öfters so genannt ist, so soll damit nicht gesagt sein, daß Lukas, der Reisebegleiter des Paulus, wirklich der Autor dieses Werkes gewesen sei. Den Versuch Harnacks, das Urteil der kritischen Forschung an diesem Punkte zu revidieren, baltc ich nicht für gelungen. 
zu reden. Der Versuch, die Stephanusrede als ein Erzeugnis 'des Lukas zu verstehen, erscheint unter allen Umständen als derjenige Weg, der zuerst begangen werden muß; erst wenn er nicht zum Ziele führt, wird man die Frage nach anderen Erklärungsmöglichkeiten aufwerfen dürfen.

Auch allen Quellenscheidungs- und Überarbeitungshypothesen gegenüber wird sich der Exeget zunächst zurückhaltend verhalten müssen. Gegeben ist uns die Rede als einheitliches Ganzes und ist als solches zunächst $z u$ verstehen. Nur dann, wenn dieses Verständnis auf unüberwindliche Schwierigkeiten stöBt, darf man zu solchen Hypothesen greifen, und auch dann muß man sich vergegenwärtigen, daß man sich auf einen sehr schwankenden Boden begibt, auf dem sich nur schwer feste Resultate gewinnen lassen. Möglichkeiten sind keine Wahrscheinlichkeiten und Wahrscheinlichkeiten keine sicheren Ergebnisse, auf denen man weiterbauen kann. Auf diè "Versuche der Kritiker, auf diesem Wege zu einer Lösung der Probleme zu kommen, kann hier nicht näher eingegangen werden; sie haben meines Erachtens wenig Überzeugendes an sich ${ }^{1}$. Denn wenn auch ein $z$ wingender Beweis für die Einheitlichkeit der Rede aus sprachlichen Gründen nicht erbracht werden kann, so läßt sich doch zeigen, daß die lukanische Eigenart auch nách der sprachlichen Seite überall bemerkbar ist.

Von den lukanischen Besonderheiten der Rede hebe ich hervor: die Anrede 72

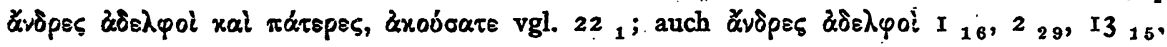
$13_{26}, 13_{38}$ und öfter. Lukanisch ist weiter der im NT nur bei. Lukas begegnende

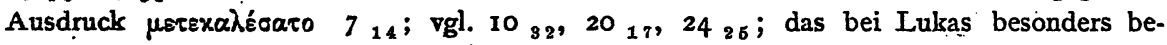

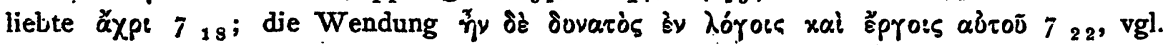
Lc 2419 ; das lukanische $\tau \tilde{\eta}$ è $\pi$ coúon $7_{26}$; vgl. $16_{11}, 20_{15}, 21_{18}, 23_{11}$; sonst nicht

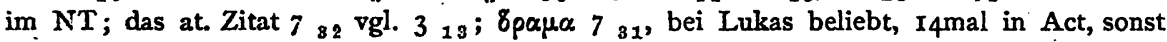

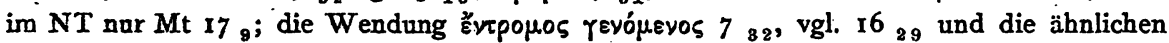

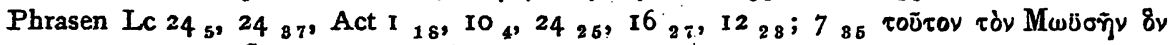

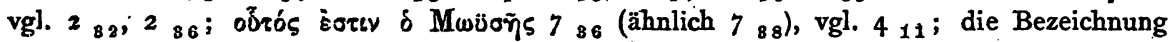
$\delta$ Bụtozos für Gott (ohne Oró́s) $7_{48}$, vgl. LC I $32, I_{35}, 6_{85}$ (gegen Mt $5_{45}$ ); zum ganzen Satz $7{ }_{48}$ vgl. $17_{24} ; 7_{52} \delta$ óixalos von Jesus absolut gebraucht, wie sonst nur noch $3_{14}, 221_{14}$. Es sei bemerkt; daß das hier vorgelegte Material auf Vollständigkeit keiren Anspruch erhebt, sondern sich noch um einiges vermehren ließe.

Wenn wir aus den angestellten Erwägungen das Recht ableiten dürfen, in der Stephanusrede ein Werk des Verfassers der Apostelakten zu sehen, so wird es für das richtige Verständnis von ent-

${ }^{1}$ Ich denke \%. B. an die. Analysen von Feine, Die vorkanonische Überlieferung des Lukas in Evangelium und Apostelgeschichte (189I) S. 186ff. und Jüngst, Die Quellen der Apostelgeschichte (1895) S. 67 ff. Feine hat sich übrigens $\mathrm{RE}^{3} \mathrm{XIX}$, 1907, S. 7 anders geäußert. 
scheidender Bedeutung sein, ob sich ermitteln läßt, welchen Zweck der Autor mit dieser Rede verfolgt.

$\mathrm{Dab}$ Lukas keinen Versuch gemacht hat, sich in die geschichtliche Situation des Stephanus hineinzudenken, fällt jedem aufmerksamen Leser auf und wird uns bei der Exegese vollends deutlich werden. Wir werden mithin von dieser Situation zunächst absehen können. Dann aber wird es, so könnte man meinen, nahe liegen, aus dem Plan der Apostelgeschichte den Gedankenzusammenhang der Rede zu erklären.

Von neueren Auslegern hat Preuschen in seinem Kommentar diesen Weg einzuschlagen versucht ${ }^{1}$. Er meint, die Rede sei dem Nachweis gewidmet, $\gg$ da: das jüdische Volk trotz der großen Wohltaten Gottes stets undankbar war und daher sein Anrecht auf Bevorzugung verscherzt hat «. Die Rede bereite so den Übergang von der Juden- zur Heidenmission vor. Indessen läßt sich gegen diese Auffassung einwenden, daß sich der erste Teil der Ausführungen, der von der. Patriarchenzeit handelt, nicht unter diesen Gesichtspunkt bringen läßt. Der Anfang der Rede erscheint dann als eine überflüssige, mit der Haupttendenz des Ganzen überhaupt nicht in Zusammenhang stehende Einleitung. Und weiter: Ist es nicht überhaupt mißlich, so allgemein von einem Plan der Apostelgeschichte $z u$ reden? Gewiß schildert der Verfasser den Übergang von der Juden- zur Heidenmission, aber es ist verfehlt, diesen einen Gesichtspunkt zur einzigen, die ganze Darstellung beherrschenden Tendenz zu machen. Eine solche Fragestellung ist die Nachwirkung der Tübinger Tendenzkritik, die allzuviel bewußte Absichten da wahrzunehmen glaubt, wo es sich höchstens um eine Einseitigkeit der Darstellung handeln kann, die die selbstverständliche, dem Autor kaum zum Bewußtsein gekommene Folge seiner christlichen Parteistellung überhaupt ist. Eine andere bewußte Tendenz als die, die Entstehung der ersten christlichen Gemeinden und die Missions-wirksamkeit und Taten der Apostel zu schildern, vermag ich in dem Werke nicht $z u$ entdecken: Alles andere, die Polemik gegen das Judentum und die Apologie gegenüber der heidnischen Umgebung, sind Nebenabsichten, die gelegentlich hervortreten, aber in ihrer Bedeutung für die Gesamtanlage des Werkes nicht überschätzt werden dürfen.

Wenn dieser Weg, die Gesichtspunkte für das Verständnis der Stephanusrede zu finden, nicht zum Ziele führt, so wird man die

${ }^{1}$ Handbuch zum Neuen Testament IV, I (1912) S. 38. 
Frage etwas anders stellen müssen. Nicht nach dem Plan' der Apostelgeschichte, sondern nach den Motiven, die die Darstellung der Stephanusgeschichte beherrschen, haben wir zu suchen, wenn wir zum richtigen Verständnis der Stephanusrede kommen wollen. Auf diese Frage ist die Antwort leicht zu geben: Der Verfasser will den Prozeß eines Märtyrers schildern.

Schon Preuschen hat in seinem Kommentar den Weg zu dieser Lösung gezeigt: Er hat darauf hingewiesen, daß die Gestalt des Stephanus mit den Zügen geschildert wird, die für die altchristliche Anschauung vom Märtyrer charakteristisch sind, und dieses Material läßt sich aus den Märtyrerakten - besonders auf Grund der Nachweise von Holl und Schlatter ${ }^{1}-$ mit Leichtigkeit vermehren. Nach dieser altkirchlichen Auffassung erscheint der Märtyrer nicht nur als Träger des Geistes (Act $7{ }_{55}$ ), was schon in den Worten Jesu, wie sie sich in den synoptischen Evangelien finden, von ihm ausjgesagt wird (Mc i 3 ,-II, Mt $10_{17-20}$, Lc .2 I I2-I4, I 2 II ff., I Petr $4{ }_{14}$ ); sondern er wird himmlischer Offenbarungen gewürdigt, schaut in Gesichten die übersinnliche Welt und tritt mit dem himmlischen

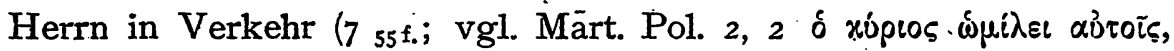
Pass. Perp. 4, 7; Mart. Pionii . 2, 2, 1 2, 3, Acta Carpi 29, 43 usw.). $\mathrm{Ja}$, der Märtyrer ist schon der diesseitigen Welt entrückt, er trägt schon die Züge der himmlischen Welt, die Mitglieder des Synedriums sehen sein Angesicht wie eines Engels Angesicht $16{ }_{15} ; \mathrm{vgl}$.

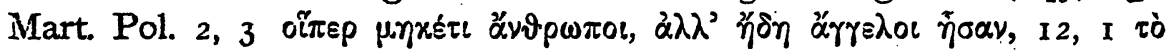

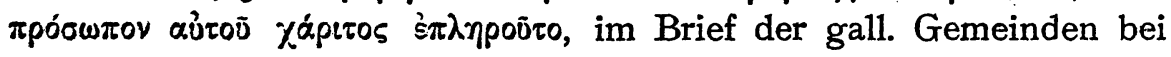
Euseb. hist. eccl. V I, 35 u. a; jüdische Parallelen: IV. Macc. 6, 2, Berachoth $9 \mathrm{a}^{\%}$ ). In grellem Kontrast dazu steht das Verhalten der

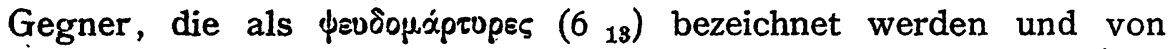
grimmigem $\mathrm{HaB}$ gegen den Märtyrer erfüllt sind ( $7 \mathrm{~s}_{4} \mathrm{ff}$ ), der sterbend noch für seine Feinde betet $(760)$.

Diese Beobachtungen dürften es nahelegen, zu versuchen, ob sich von hier aus ein besseres Verständnis der Stephanusrede ge-

1 Holl, Die Vorstellung vom Märtyrer und der Märtyrerakte in ihrer geschichtlichen Entwicklung, Ilbergs Neue Jahrbücher für das klassische Altertum XXXIII, I914, S. 52 I ff. Schlatter, Der Märtyrer in den Anfängen der christlichen Kirche (Beitr. z. Förd. christl. Theol. XIX, 3, 1915). Auf die Registrierung der weiteren Literatur, die haupt-

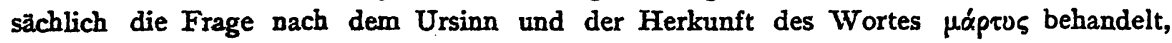
verzichte ich; doch möchte ich bemerken, daß ich mich der Auffassung Holls, der Name Märtyrer rühre daher, daß dieser von der ihm geschenkten Offenbarung aus der übersinnlichen Welt Zeugnis ablege, nicht anzuschließen vermag.

2 Vgl. Schlatter S. 36. 84; weiteres Material bei Preuschen, Kommentar S. 38. 45; Holl S. 525 f. 
winnen läßt. Auch sie ist irgendwie in den aufgezeigten Zusammenhang einzuordnen. Wir werden jedenfalls erwägen müssen, ob sie uns als eine Verteidigungsrede eines Märtyrers, die der Verfasser der Apostelgeschichte dem Stephanus in den Mund legt, verständlich werden kann.

Aber ehe wir an die Analyse der Rede herantreten, haben wir uns darüber klar $z u$ werden, an welche Adresse diese Apologie gerichtet ist. $\mathrm{Da} ß$ der Verfasser der Apostelgeschichte auf den eigentlichen Vorwurf, der gegen Stephanus erhoben ist, in dieser Rede nicht eingeht,' ist schon angedeutet worden und wird weiter unten genauer ausgeführt werden. Der Gedanke, den neuere Ausleger, wie Wendt ${ }^{1}$, darin finden, daß $»$ Gottes Heilsgegenwart nicht an den Tempel gebunden sei «, ist nirgends deutlich ausgesprochen, auch 748 nicht. Man kann es begreifen, daß Exegeten wie $\mathrm{Bla}^{2}{ }^{2}$ auf den Gedanken kommen, die Rede sei ein Torso und der Lärm habe Stephanus gehindert, seinen Gedankengang zu Ende zu führen, wenn freilich auch auf diese Weise der gordische Knoten mehr zerhauen als gelöst wird. Für den Ausleger, der in der Rede nicht die Wiedergabe der wirklich gesprochenen Worte erblickt, ist es vielleicht richtiger, die Frage aufzuwerfen, ob man dem Verfasser überhaupt soviel geschichtliches und psychologisches Verstehen zutrauen darf, daß man annehmen muß, er habe sich in die Situation des geschichtlichen Stephanus hineinversetzen können ${ }^{3}$. Wie er das Bild des Stephanus nach der Märtyrervorstellung seiner Zeit gezeichnet hat, so wird er auch keinen Unterschied zwischen den jüdischen Gegnern, mit denen er es zu tun hat, und dem Synedrium. dem Stephanus Rede und Antwort stehen muß, gemacht haben. Er läßt den Stephanus zu der jüdischen Behörde genau so reden, wie sich ein Christ seiner Zeit gegen die Angriffe der Gegner zu verteidigen pllegte. Er tut damit nichts anderes als etwa der Verfasserdes Johannesevangeliums, dem der Gedanke, daß der historische Jesus anders zu seinen Gegnern gesprochen haben könnte, als er ihn reden läßt, wohl unerschwinglich gewesen wäre, ebenso unfaßlich, wie überhaupt einem Christen der nachapostolischen Zeit die Idee; $\mathrm{da} ß$ das Christentum sich entwickelt babe und daß die werdende katholische Kirche nicht mehr diejenige cler apostolischen Zeit sei. Es handelt sich ja auch nicht um Geschichtsfälschung, wenn Lukas

1 Kommentar S. 138.

$2 \mathrm{Bla \beta}$, Acta apostolorum, editio philologica, 1895, S. 104.

$3 \mathrm{Vgl}$. auch die Bemerkungen von Wendland, Hellenist.-röm Kultur ${ }^{2}$ (I9I2) 33 If. 
die Verhältnisse seiner Zeit in das apostolische Zeitalter zurückìträgt $\left(\begin{array}{l}6_{\text {rif. }} \\ 1\end{array} 4_{23}\right)$, sondern es kommt hier nur der Mangel an geschichtlichem Denken. zum Ausdruck, zu dem der Mensch ja erst auf einer Stufe entwickelter Reflexion befähigt ist.

Das heibt mit anderen Worten: Die Rede, die Stephanus an das. Synedrium richtet, ist in Wirklichkeit eine apologetische Ansprache, in der sich der Verfasser der Apostelgeschichte an die Gegner des Christentums seiner Zeit richtet, und zwar denkt er in erster Linie an die Juden, die ja in der Apostelgeschichte - ähnlich wie im Johannesevangelium, aber auch z. B. im Martyrium des Polykarp $(12,2.13,1.17,2,18,1)$ - als diejenigen erscheinen, von denen der erbittertste Widerstand gegen die Christen ausgeht (vgl. Act I3 $50,142.19,175.13$ ) und die als die eigentlichen Feinde der neuen Religion zu gelten haben.

Der Mangel an geschichtlicher Denkweise - auch auf diesen Punkt muß noch mit einigen Worten eingegangen werden - zeigt sich aber auch bei der Verwertung des AT. Auch das ist eigentlich selbstverständlich, denn eine religiöse Urkunde, die als kanonische Autorität absolute Geltung beansprucht, kann ihrem Wesen nach nicht geschichtlich verstanden werden. Denn sie wird ja von den Frommen nicht als Dokument bestimmter geschichtlicher Verhältnisse, sondern als die geoffenbarte Norm von übergeschichtlicher, bleibender Bedeutung angesehen, die dem Frommen auf alle Fragen Antwort geben muB, die sein Herz bewegen. Den Christen der ersten Zeit ist es ganz selbstverständlich gewesen, daß das AT und die Propheten keine andere Bedeutung gehabt haben, als daß sie Zeugen und Verkündiger Christi und der messianischen Zeit gewesen sind. Wird diese Auffassung bewußt durchgeführt und zu exegetischer Methode ausgebildet, so kommt man zu einer Art typologischer oder allegorischer Exegese; aber auch da, wo man nicht mit solcher bewußten Methode arbeitet, steht man den heiligen Texten mit ähnlicher Einstellung gegenüber; ganz. unwillkürlich liest der Gläubige seine eigenen Fragen und Probleme in sie hinein. So hat Luther und der Aitprotestantismus die Bibel lediglich unter dem einen Gesichtswinkel Sünde und Gnade betrachtet und dem entsprechend in Gesetz und Evangelium geteilt. Etwas Ähnliches gilt aber auch von dem Verfasser der Apostelgeschichte, der ja auch von dem messianischen Weissagungsbeweis Gebrauch gemacht hat 2 25 ff. $3{ }_{22}$ ff. usw.). Auch er ist selbstverständlich davon überzeugt, daß die at. Frommen im wesentlichen die Züge der Christen seiner Zeit getragen haben und dab die Verfolgung der Frommen durch 
die Gottlosen, die jetzt die Christen erleben, sich in der Väter Tagen genau so zugetragen hat wie in der Gegenwart. Dalo aber die Christenheit das Recht hat, sich den Juden gegenüber auf das AT zu berufen, ist ihm wohl keinen Moment zweifelhaft gewesen. Er hat das AT nicht mit der Einstellung des modernen Historikers gelesen, und dieser hat, wenn er seinen Gedankengängen folgen will, seine eigene historische Einstellung dem AT gegenüber vollkommen auszuschalten. Nur wenn dieser Gesichtspunkt hinreichend beachtet wird, wird es uns möglich sein, zu einem befriedigenden Verständnis der Rede zu gelangen.

Die vorangegangenen Erörterungen sollten dazu dienen, die für das' Verständnis der Perikope notwendigen Voraussetzungen zu gewinnen. Die Analyse selbst wird nunmehr zu zeigen haben, ob sich der Gedankenzusammenhang der Rede von hier aus wirklich begreifen läßt. Ihr haben wir uns nunmehr zuzuwenden.

Die Rede beginnt mit einem Rückblick auf das Leben Abrahams (2-8). Der Redner hebt hervor, daß Abraham zweimal von Gott in ein anderes Land geführt worden sei, einmal von Mesopotamien nach Haran und von dort in das Land, in dem die Juden jetzt wohnen (3. 4), nach Palästina. Auch in diesem Land hat ihm Gott keine dauernde Heimat gegeben, nicht einen Fußbreit Landes hat Abraham besessen; nur die Verheiloung ward ihm zuteil, $\mathrm{da} B$ er und sein Same es einstmals in Besitz nehmen würden (V. 5). Aber auch diesem Samen hat Gott kein anderes Los in Aussicht gestellt, auch er wird ein Beisasse in fremdem Lande sein. Der Zweck dieser Ausführungen kann nicht verkannt werden, er hat freilich nichts mit der Situation des geschichtlichen Stephanus zu tun und auch zum Tempelkult gar keine Beziehung: A braham und sein Same sind ebenso $\pi$ d po $x$ oc in fremdem Lande gewesen, wie heute die Christen $\pi \alpha$ porxol sind. Denn die Christenheit ist ja das Volk der Verheißung, der wahre Same Abrahams ( $R m{ }_{18}$ ); und was von ihnen gilt, das hat schon vom at. Bundesvolk und dem Erzvater Abraham gegolten.

Die Vorstellung, daß die Christen Fremdlinge auf Erden, heimatlose Pilger sind, die hier keine bleibende Statt haben (Hebr $13{ }_{14}$ ), ist bekannt und kann durch viele Zeugnisse urchristlicher und altkirchlicher Frommigkeit belegt werden; für den Zusammenhang unserer Untersuchung ist wichtig, hervorzuheben, daß sie sich gerade in Märtyrerakten, so in den Zuschriften des Polykarpmartyriums oder des Briefes der gallischen Gemeinden (Eus. hist. eccl. V, I, 13) 
nicht selten findet; und ebenso ist es nicht zufällig, daß auch der erste Petrusbrief, ein Schreiben ${ }^{1}$, das an Gemeinden gerichtet ist, die unter dem Druck heidnischer Bevölkerung stehen und von. Verfolgungen zum mindestein bedroht sind, diese Seite des Christentums besonders hervorhebt (I Petr $I_{1}$. $2_{11}$ ). Auch eine apologetische Schrift wie der Brief an Diognet $(5,5 ; 5,9$, vgl. überhaupt den ganzen Abschnitt Kap. 5 und 6) wäre hier zu erwähnen. Es ist ja ohne weiteres psychologisch begreiflich, wie gerade angesichts der vielen Anfeindungen von heidnischer oder jüdischer Seite dies Gefühl der Fremdheit, der Zugehörigkeit $z u$ einer andern Welt bei den Christen immer neue Nahrung erhielt und auf der andern Seite ihnen zum Vorwurf gemacht wurde; aus der jenseitigen, der übersinnlichen Welt zugekehrten Einstellung; der Christen und ihrer eschatologischen Zukunftshoffnung wurde von christenfeindlicher Seite der Grund zu der Beschuldigung des odium generis humani (Tacitus, ann. XV, 44) gefünden. Gegen diese Anklage wendet sich der Eingang der Stephanusrede: dieser. Vorwurf ist unberechtigt, von jeher ist diese Heimatlosigkeit die Eigenschaft der Gotteskinder gewesen, schon die Frommen des alten Bundes, schon Abraham und sein Same sind heimatlose Fremdlinge auf Erden gewesen, die auf die Verheißung Gottes gehofft. haben. Jüdischen und heidnischen Gegnern mögen solche Ausführungen wenig überzeugend erschienen sein; den christlichen Lesern werden sie sehr eingeleuchtet haben.

Auch die Fortsetzung der Rede $6_{6} \cdot 7$ erscheint von hier aus unmittelbar verständlich. Die Bedrückung des Samens Abrahams entspricht durchaus dem, was die Christen jetzt $z u$ erdulden haben; den Feinden aber, die das Gottesvolk bedrücken, steht Gottes Strafgericht ebenso bevor, wie den Auserwählten selbst die Erlösung. Auch hier findet sich in den Märtyrerakten eine interessante Parallele; in der Passio Perpetuae XVII erinnern die Märtyrer die Heiden an das kommende Gericht; der Märtyrer Saturus sagt sogar mit kräftigem Realismus: notate tamen vobis facies nostras diligenter, ut recognoscatis nos in die illo2. Durch solchen Hinweis auf das Gericht sollen die Gegner des Christentums zur Einkehr und Buße

1 Die Hypothese, daß der erste Petrusbrief ursprünglich in seinem wesentlichen Bestande eine Taufhomilie gewesen sei, für die Perdelwitz (Rel. Vers. u. Vorarb. XI, 3, I9II/r2) und Bornemann, Zeitschr. f. neutest. Wiss. XIX, 1920, S. 143 ff. eingetreten sind, entbebrt meines Erachtens genügender exegetischer Grundlagen. Die Deutung der dafür geltend gemachten Stellen ist keineswegs sicher.

2 Knopf, Ausgewählte Märtyrerakten (1913) S. 5 I. 
gebracht werden; ein Erfolg, der ja in vielen Fällen (vgl. z. B. den Schluß von Kap. XVII der Pass. Perpet.) auch erreicht worden ist.

$\mathrm{Ob}$ bei der Erwähnung der Beschneidung (V. 8) dem Verfasser eine typologische Beziehung auf die Taufe vorgeschwebt hat, will ich nicht sicher entscheiden, um mich nicht dem Vorwurf auszusetzen, als lege ich $z u$ viel in die Rede hinein; vielleicht soll dieser Vers nur überleiten zu dem $z$ weiten Beispiel der at. Geschichte, dem Beispiel Josephs. Auch hier ist der apologetische Zweck der Ausführungen nicht $\mathrm{zu}$ verkennen: Joseph erscheint als Typus des Frommen, der von der Welt gehaßt und verfolgt wird, sich aber Gottes wunderbaren Schutzes erfreuen darf und seinen. Brüdern Böses mit Gutem vergilt. Dasselbe haben auch die Christen geflissentlich ihren Gegnern gegenüber hervorgehoben; wie der erste Petrusbrief seinen Lesern einschärft; daß sie durch ihren Wandel die Gegner beschämen sollen (2 ${ }_{12} .3_{16}$, vgl. auch Ign. ad Eph. I0, 2), so schildert auch der Brief an Diognet die Christen als die unschuldig

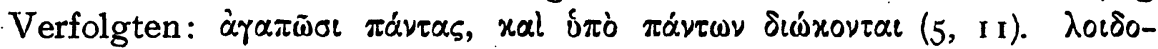

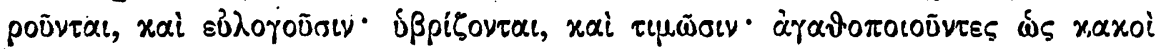

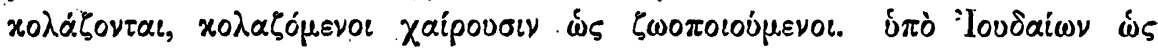

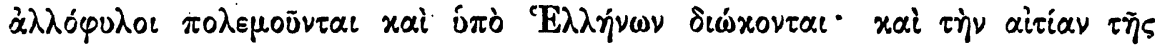

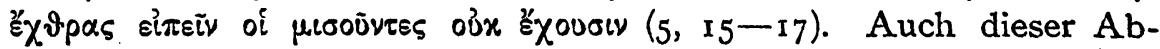
schnitt scheint am Schluß einen Hinweis auf die $\pi \dot{\alpha}$ porxia der Christen zu enthalten; wenigstens legt die ausdrückliche Erwähnung des Todes der Erzväter in Ägypten und ihre Bestattung in dem Grabe, das Abraham in fremdem Lande gekauft hatte, ein solches Verständnis nahe (V. I5, I6).

V. I7 ff. wendet sich der Mosegeschichte zu. Auch hier wird wieder, wie schon vorhin (V. 6), die Bedrückung des auserwählten Volkes durch Pharao hervorgehoben, die Bedrückung, die der Zeit

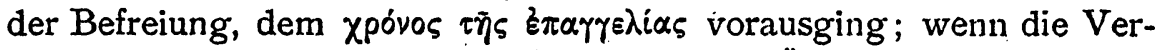
mehrung und Ausbreitung des Volkes in Ägypten ausdrücklich hervorgehoben wird, so liegt es nahe, darin einen Hinweis auf die rasche Ausbreitung des Christentums im nachapostolischen Zeitalter $z u$ sehen, wie sie uns beispielsweise durch den Brief des Plinius (Ep. X, 96) bezeugt wird, auch der erste Petrusbrief ist ja vielleicht an Gemeinden, die in ihrer Mehrzahl aus Neophyten bestehen, gerichtet ${ }^{1}$. Es ist deshalb nicht nötig, anzunehmen, daß die Apostelgeschichte in Kleinasien geschrieben ist; denn solches schnelles Wachstum der Gemeinden ist auch in anderen Gegenden nicht un-

1 Vgl. Knopf (in Meyers Kommentar ${ }^{7}$ 1912) zu I Petr $2_{2}$. 
denkbar. Daran, daß hier die Israeliten als die Bedrückten erscheinen, während sie im weiteren Verlaufe der Rede als diejenigen geschildert werden, die dem Friedebringer und Erlöser Moses verständnislos und ablehnend gegenüberstehen, werden wir bei der Art der Schriftbehandlung unseres Autors keinen Anstoß nehmen dürfen. Der Exeget wird solche Inkonzinnitäten ruhig in den Kauf nehmen, sofern ihm nur der Gedankengang psychologisch begreiflich erscheint.

Die Erwähnung der Bedrückung des auserwählten Volkes in Ägypten ist freilich für den Verfasser nur ein Nebengedanke, der zu dem dritten, ausführlichsten Beispiel überleitet, der Geschichte des Moses. Bei diesem Paradigma verweilt der Redner mit besonderer Vorliebe und die Gesichtspunkte verschieben sich ihm allmählich. Zunächst hat man den Eindruck, auch Moses sei nichts anderes als ein Typus des Frommen - auch bei ihm wird sein Beruf zum Friedestifter (V. 26) und seine Heimatlosigkeit hervorgèhoḅen (V. 29) - aber in der zweiten Hälfte der Darlegungen (von V. $30 \mathrm{ab}$ ) wird es ganz deutlich, daß Moses nicht nur der Typus des von den Gottlosen bedrückten Frommen, sondern des Messias überhaupt ist. Diese Verschiebung der Gesichtspunkte ist vom psychologischen Standpunkte aus nicht schwer zu begreifen, denn dem Messias ist kein anderes Schicksal widerfahren als dasjenige, das den verfolgten und angefeindeten Christen immer wieder zuteil wird. Mit dieser Wendung des Gedankens hängt aber noch ein Zweites zusammen; mit dem Gedanken an Christus verbindet sich für den Verfasser sofort der Gedanke an das ungläubige Judentum, das den Messias von sich gestoßen hat. So kommt es, daß die Rede von der Verteidigung, die in dem ersten Teil weitaus vorwiegt, zum direkten Angriff gegen die Hauptgegner des Christentums, die Juden übergeht.

Auch dieser Übergang von der Apologie der eigenen Religion zu einer energischen Polemik gegen ihre Hauptfeinde wird uns nach dem Gesagten nicht auffallend erscheinen können; und diese Polemik beherrscht die weiteren Ausführungen bis zum Schluß und wird gegen Ende der Rede zusehends schärfer. Nicht nur, daß die Juden Moses, den ihnen von Gott zum Erlöser und Führer bestimmten Gottesmann, in Ägypten zurückgewiesen haben $\left(\begin{array}{l}73 \\ 35\end{array}\right)$, sie

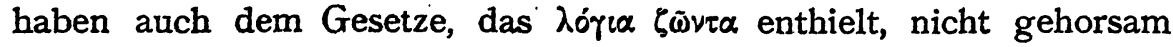
sein wollen, sondern durch ihren Götzendienst sich aufs neue schwer versündigt ( 7 g ff.). Aber nicht nur ihr Götzendienst in der Wüste, sondern auch der Tempelbau selbst wird als Abfall von Gott gebrandmarkt; denn die Verse 44-46 können kaum anders als in dem 
Sinne verstanden werden, daß zwar die Stiftshütte in der Wüste nach göttlicher Weisung erbaut worden sei, daß aber der Tempelbau Salomos einen Abfall von dem wahren Gottesdienst bedeute: das

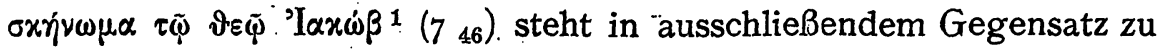
dem oixos (V. 47), das Salomo Gott erbaut hat. Denn der Gedanke, daß der Höchste nicht in Werken wohne, die von Menschenhänden gemacht sind, bedeutet doch hier wie ${ }_{7}{ }_{24}$ eine Ablehnung des Tempelkultes ohne irgendwelche Restriktionen. Die nächste Parallele zu unserer Stelle bietet in der Tat der Barnabasbrief, der ebenfalls den Tempelkultus völlig verwirft und in Kap. 16, 2 dieselbe Jesajastelle $\left(66\right.$ x.) als Schriftbeleg verwertet ${ }^{2}$; diese Parallele legt es auch nahe,

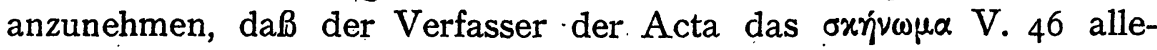
gorisch auf die Wohnung Gottes im Herzen gedeutet habe (vgl. Barn. 16, $6 \mathrm{ff}$.): David fand Gnade bei Gott und bat, daß er in seinem Innern eine Wohnung für Gott finden möge. Diese Deutung ist freilich nur ein Versuch, der schwierigen Stelle Herr zu werden; aber wie man auch über sie urteilen mag, den Gegensatz zwischen V. 46 und 47 wird man nicht verkennen können. Dem frommen David wird der von Gott abgefallene Salomo entgegengestellt, der Tempelbau als etwas dem göttlichen Willen Entgegengesetztes bezeichnet. Bei diesem Verständnis erscheinen die zusammenfassenden Verse $50-53$ nicht als ein abrupter Übergang $\mathrm{zu}$ einem anderen Punkte, sondern als eine kurze Zusammenfassung der Vorwürfe, die gegen die Juden erhoben werden; stets haben diese dem heiligen Geiste widerstrebt, stets die Propheten, die wie Mose (V. 37) das Kommen des Gerechten geweissagt,haben, verfolgt; auch das Gesetz, das Moses im Auftrag der Engel überliefert hat, haben sie nicht gehalten. Mit diesen wuchtigen Sätzen schließt die Rede, die nun, wie zu erwarten ist, die Wut der Juden aufs höchste steigert.

Wenn wir auf den Gedankengang zurückblicken, so müssen wir sagen, daß es verfehlt wäre, die Rede als ein rhetorisches, streng logisch zusammenhängendes Kunstwerk zu bezeichnen. Aber wir haben auch nicht das Recht, mit allzu hohen Anforderungen in dieser Beziehung an den Verfasser der Apostelgeschichte heranzutreten, und die Exegese ist nicht verpflichtet, logischer als der Text selbst zu sein, sondern muß sich damit begnügen, den Gedanken-

${ }^{2}$ Die Lesart okxy wird mit den meisten neueren Exegeten trotz guter Bezeugung als sinnlos abzulehnen sein.

2 Ich sehe nachträglich, daß Windisch in seinem Kommentar zum Barnabasbrief (Handbuch zum NT Ergänzungs-Bd. III), S. 394 f. auf diese Verwandtscbaft schon hingewiesen hat.

24. 6. 1922 
gängen des Autors nachzugehen und sie in ihren Motivationszusammenhängen zu erfassen. Und aufs Ganze gesehen, erscheinen diese uns durchsichtig und, dem Zweck des Lukas entsprechend, durchaus verständlich. Dem Christen jener Zeit wird das Verständnis der Rede keine Schwierigkeiten bereitet haben. Der Zusammenhang mit der apologetisch-polemischen Gesamthaltung der Apostelgeschichte, die die Schuld der Juden an den Christenverfolgungen immer und immer wieder hervorhebt, die Christen dagegen als die unschuldig und unrechtmäßig. Verfolgten hinstellt, ist nicht $\mathrm{zu}$ verkennen. So ist durch die Exegese der Beweis dafür, daß wir es hier nicht mit einer Ansprache des historischen Stephanus, sondern einem Werke des Lukas zu tun haben, erbracht worden.

Jene Annahme darf in der Tat, wenn unsere Auffassung richtig ist, als völlig ausgeschlossen gelten. Eine derartige Polemik gegen das Judentum wäre für einen Juden selbst, der sich noch in irgendeiner. Weise zu seinem Volk gehörig fühlte, unmöglich gewesen. Auch Paulus hätte solche Sätze niemals schreiben können. Für die Annahme, daß wir es hier mit einem vorpaulinischen Typus des Christentums z丩 tun hätten, finden sich keinerlei Anlialtspunkte. Im Gegenteil: die paulinische Fragestellung »Gesetz oder Glaube « ist bereits aus dem Gesichtskreis des Verfassers geschwunden. Das Gesetz ist ihm nicht mehr, wie dem Paulus, eine Willensäußerung Gottes, die zeitweilige Geltung beanspruchen konnte, nun aber durch eine andere Kundgebung Gottes außer Kraft gesetzt ist. Es wird

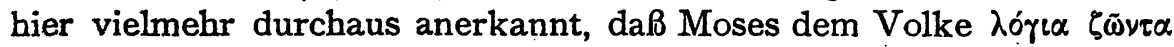
gegeben habe (V. 38); aber die Juden haben dieses Gesetz nicht gehalten. Die rationalistische Polemik gegen den Tempelkultus, die in der hellenistischen Aufklärung mancherlei Parallelen hat ${ }^{1}$, zeigt, daß der Verfasser, wenn er von dem Gesetz redet, schwerlich an das Zeremonialgesetz gedacht haben wird. Auch die Apologetik im ersten Teil der Rede, der Versuch, die Heimatlosigkeit und das Himmelsbürgertum der Christen durch den Hinweis auf die at. Frommen zu rechtfertigen, macht die Entstehung der Rede in einer Zeit wahrscheinlich, in der die neue Religion schon die Augen der Welt auf sich zieht.

Wenn wir die gegen Stephanus erhobene Anklage aus 614 entnehmen dürfen, so sehen wir, daß sie sich auf einen anderen Punkt bezieht. In diesem Wort ist von einer völligen Verwerfung

1 Vgl. jetzt Windisch zu Bam. 16, 4; dazu das schon von Wetstein zitierte Fragment Ps. Euripides fr. 1130 (s. Preuschen 2u Act $7{ }_{48}$ ).

Zeitschr. f. d. pentest. Wiss. 20. Band $192 x$. 
des Tempelkultus nicht die Rede. Für die Gegenwart wird die Geltung des Gesetzes und das Recht des Tempelkultus nicht in Frage gestellt. Es wird nur gesagt, daß Jesus, naürlich bei der Parusie, den Tempel zerstören und die Geltung des Gesetzes außer Kraft setzen, d. h. eine neue Ordnung im messianischen Reiche auf-. richten werde. Vielleicht hat Stephanus an das Wort Jesu von der Zerstörung des Tempels, das nach Markus und Matthäus bei dem Prozeß Jesu eine Rolle gespielt hat und möglicherweise den Grund zur Verurteilung Jesu abgegeben hat - die Evangelisten stehen ihm mit ziemlicher Verlegenheit gegenüber ${ }^{1}$ - wieder angeknüpft ${ }^{2}$. Für die Ohren strenggläubiger Juden sind solche Äußerungen freilich kaum eine geringere Blasphemie gewesen als der Radikalismus des Paulus mit seiner völligen Negierung des Gesetzes. Es dürfte deshalb auch kaum ein Zufall sein, daß aus dem Kreise des Stephanus - denn dieser, nicht die ganze Urgemeinde wird nach dem Tode des ersten Märtyrers geflohen sein - die ersten Heidenmissionare hervorgegangen sind (Act I I ${ }_{9} \mathrm{ff}_{\text {. }}$ ).

Im übrigen ist die Gestalt des Stephanus, wenn wir die Rede nicht für die Rekonstruktion seiner Anschauungen verwerten dürfen, für uns in ziemliches Dunkel gehüllt. Wir können nicht mehr von ihm sagen, als daß er eine der führenden Persönlichkeiten des Siebenerkollegiums gewesen ${ }^{8}$ und wegen einer radikalen Äußerung über das Gesetz und den Tempel das Opfer eines jüdischen Pogroms geworden ist. Die ausführliche Schilderung des Martyriums ist dem Verfasser der Apostelgeschichte aufs Konto zu setzen, dem wir ja auch die eingelegte Rede zuzuschreiben haben. Aber gerade die Diskrepanz zwischen der erhobenen Anklage und der Rede zeigt, daß der Verfasser von einer Tradition abhängig sein wird. Auch sonst haben wir in den Kap. $6-8_{4}$ Spuren, die auf eine Quellenbenutzung hinweisen. Die auffallenden Wiederholungen $6_{11}$ und $6_{13}, 7_{55}$ und ${ }_{56}$ und das unvermittelte Auftreten des Saulus $758 \mathrm{~b}$, $8_{1}$ und $8_{3}$ legen die Annahme einer Quellenbearbeitung nicht minder nahe wie die Tatsache, daß die ganze Darstellung zwischen der Schilderung einer regelrechten Gerichtsverhandlung und eines

${ }_{1}$ Nach MC ${ }_{4}{ }_{58}$, MIt $24_{61}$ handelt es sich um eine Verleurndung falscher Zeugen, bei Lukas fehlt das Wort, nach Johannes 2 igff. ișt es bei der Tempelreinigung gesprochen und allegorisch zu deuten.

2 Stephanus reißt . . . eine Wunde wieder auf, welche dic Urjünger gerne vernarbt gesehen hätten« (Wellhausen, Nachr. d. Götting. Ges. d. Wiss. 1907, S. I 2).

- Ich sehe mit E. Schwartz (Nachr. d. Götting. Ges. d. Wiss. 1907, S. 280 ff.) in diesem Kollegium den Missionsausschuß der bellenistischen Kreise der Urgemeinde. 
gewaltsamen Pogroms hin und her schwankt. Wir werden das letztere für das Ursprüngliche und die Gerichtsverhandlung auf das Konto des Lukas zu setzen haben, der so für seine Apologetik und Polemik den nötigen Raum gewinnt. Eine bis ins einzelne durchgeführte Scheidung dessen, was Lukas aus der Quelle übernommen hat und was sein eigener Zusatz ist, ist nicht durchführbar. $\mathrm{Zu}$ sicheren Ergebnissen wird es sich wenigstens bei solchen Versuchen schwerlich bringen lassen.

Indessen das Ergebnis unserer Untersuchung ist nicht schlechthin negativ. Die Eigenart des Verfassers der Acta, seine apologetischen und polemischen Tendenzen sind durch sie deutlicher geworden. Wir gewinnen aus der Stephanusgeschichte ein lebendiges Bild von der Auseinandersetzung des Christentums mit seinen jüdischen Gegnern, eine Anschauung von dem Gegensatz der Christen zu der sie umgebenden Welt. Wir sehen hier die weltäbgewandte, dem Jenseits zugekehrte Stimmung. der alten Christen, die sich als heimatlose Fremdlinge auf Erden fühlen, und die die negative Kèhrseite des Enthusiasmus bildet, mit dem sie ihre Märtyrer verehrten, die durch ihr Leiden himmlischer Gesichte teilhaftig und mit dem heiligen Geiste ausgerüstet werden. Wir sehen auch das hohe Selbstbewußtsein der Christen in dieser Urkunde sich widerspiegeln, mit dem sie, die Verfolgten und Gehaßten, sich als das wahre Gottesvolk, die Erben der Verheißungen, die Friedestifter in der Welt fühlen. Wenn wir mit solchen Augen diese kleine Perikope betrachten, so kann auch sie uns einen wertvollen Beitrag zu dem höchsten und letzten Ziele der nt. Wissenschaft liefern, einen Beitrag zu dem Verständnis lebendiger urchristlicher Frömmigkeit.

[Abgeschlossen am 11. Juni 1921.]

\section{Englisch-amerikanische Literatur zum Neuen Testament in den Jahren 1914-1920.}

Von Hans Windisch in Leiden.

II.

Zur Apg. hat der amerikanische Orientalist C. Torrey einen sehr wichtigen Beitrag geliefert: *The Composition and Date of Acts ${ }^{1}$. Die wichtigste These ist die, daß die ganze erste Hälfte

1 Harv. Theol. Studies I Cambridge (Mass.) 1916. 72 p. (z. Z. vergriffen). 